'Departamento de Odontología Restauradora, Facultad de Odontología, Universidad de Concepción, Concepción, Chile. ${ }^{2}$ Departamento de Educación Médica, Facultad de Medicina, Universidad de Concepción, Concepción, Chile.

${ }^{3}$ Carrera de Psicología Sede Concepción, Facultad de Psicología, Universidad San Sebastián, Concepción, Chile. ${ }^{4}$ Unidad de Investigación, Enfoque Émico, Concepción, Chile.

5Oficina de Educación Médica, Facultad de Medicina, Universidad Católica del Norte, Coquimbo, Chile. ${ }^{a}$ Cirujano dentista, Magíster (c) en Educación Médica para Ciencias de la Salud. bPsicólogo, Magíster en Psicología con mención en Psicología Educativa.

'Psicóloga, Magíster en Ciencias de la Educación.

${ }^{d}$ Ingeniero ( $E$ ) en Administración. 'Magíster en Educación Médica para las Ciencias de la Salud.

Trabajo financiado por el proyecto FONDECYT Regular 1161541.

Los autores no declaran conflicto de intereses.

Recibido el 30 de diciembre de 2016, aceptado el 3 de mayo de 2017.

Correspondencia a: Diego Antonio Troncoso Gacitúa Facultad de Odontología Universidad de Concepción Avenida Roosevelt 1550 Concepción, Chile. dtroncos@udec.cl

\section{¿Se relaciona la capacitación docente con las prácticas pedagógicas en académicos de carreras de la salud de Chile?}

\author{
DIEGO TRONCOSO G. ${ }^{1, a}$, CRISTHIAN PÉREZ V., ${ }^{2, b}$, \\ GIULIETTA VACCAREZZA G. ${ }^{3, \mathrm{c}}$, CÉSAR AGUILAR A. ${ }^{4, \mathrm{~d}}$, \\ NADIA MUÑOZ N. ${ }^{5, e}$
}

\section{The influence of pedagogic and discipline training on the teaching quality of university professors}

Background: University teachers prioritize acquiring knowledge about their disciplines over pedagogic training. However, the latter is becoming increasingly important in the present teaching scenario. Aim: To relate pedagogic practices with pedagogic training of teachers from health care careers of public and private universities. Material and Methods: Pedagogic practice and training activities participation questionnaires were answered by 296 teachers of undergraduate students from Chilean public and private universities. Results: There was a direct correlation between discipline training and all pedagogic practice factors. However, pedagogic training correlated with all the factors with the exception of teacher centered learning. Teachers with a master degree had higher scores in factors related to teaching planning and process assessment. Having a doctor degree had no impact on these factors. A multiple regression analysis showed that both discipline and pedagogic training and having a master degree were associated with pedagogic practices of teachers. Conclusions: Both pedagogic and discipline training influence the quality of teaching provided by undergraduate teachers.

(Rev Med Chile 2017; 145: 610-618)

Key words: Education, Medical; Faculty, Medical; Programmed Instruction as Topic; Staff Development; Teaching.

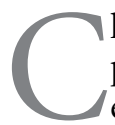
hile, como el resto de Latinoamérica, presenta un crecimiento explosivo de su educación superior, con un incremento abrupto del número de universidades, carreras ofrecidas y matriculados ${ }^{1-2}$.

Esta explosión derivó en un alumnado más diverso ${ }^{3}$ y una mayor demanda por profesionales que asuman el rol docente ${ }^{1}$. A esto, se suman los cambios en los modelos educativos, que ponen al estudiante como protagonista del proceso formativo, exigiendo al docente que deje atrás su rol tradicional de transmisor de información y se vuelva un facilitador del aprendizaje del estudiante ${ }^{4-7}$.

Estos cambios han aumentado la presión sobre el rol del docente, volviendo fundamental cuestionarse por las prácticas pedagógicas de éste. Sobre todo, porque el docente universitario no suele ser un profesional de la educación y su elección está más asociada a su experticia disciplinar, no pedagógica ${ }^{8}$.

Las prácticas pedagógicas no son sólo las actividades que se desarrollan en aulas o laboratorios, 
orientada por un currículo a fin de formar estudiantes ${ }^{9}$, sino que se refieren a los procesos donde los docentes reflexionan y analizan aspectos, tanto intelectuales como afectivos, de las instancias educativas, para definir cómo implementarán la enseñanza ${ }^{10-12}$.

La complejidad de dichas prácticas se contrapone al carácter antes mencionado de la docencia universitaria, que algunos consideran "preprofesional" o "semiprofesional", pues se basaría en métodos aprendidos de manera artesanal o vicaria $^{13}$.

Bajo la premisa que un académico puede ser especialista en tema, pero no contar con las competencias pedagógicas necesarias para enseñarlo ${ }^{14}$, es que en la actualidad existen esfuerzos sostenidos de parte de las universidades para capacitar a sus docentes, tanto en temáticas propias de la disciplina $^{15}$ como educativas ${ }^{15,16}$. Esto, pues se asume que la formación pedagógica es necesaria para un ejercicio más eficiente de la labor educativa, aunque esto necesita también un profundo dominio de la especialidad que se imparte ${ }^{17,18}$.

Aunque las instancias de capacitación, tanto disciplinares como pedagógicas, son valoradas positivamente por los docentes ${ }^{15,16}$, existe poca evidencia de su impacto en el aula. En Chile, se ha observado que el número de docentes con doctorado y magíster se asocia a menores tasas de deserción ${ }^{1}$ y que los docentes tienen una percepción positiva del efecto de las capacitaciones educativas $^{15,16}$. Sin embargo, no existen estudios que documenten la relación entre perfeccionamiento y quehacer docente. Por este motivo, el presente estudio busca relacionar la participación de los docentes de carreras de la salud en actividades de perfeccionamiento disciplinar y pedagógico con las prácticas pedagógicas de estos.

\section{Método}

\section{Participantes}

El estudio se realizó con profesionales que imparten docencia de pregrado en carreras de la salud en universidades chilenas, tanto tradicionales (adscritas al Consejo de Rectores de Universidades Chilenas, CRUCH) y privadas (no adscritas al CRUCH).

Se seleccionó una muestra de 296 participantes mediante muestreo por voluntarios (Tabla 1).

\section{Instrumentos}

Los participantes respondieron tres cuestionarios:

Tabla 1. Descripción de la muestra de docentes de carreras de la salud

\begin{tabular}{|c|c|c|}
\hline Variable & \multicolumn{2}{|l|}{ Estadísticos descriptivos } \\
\hline Edad (en años) & \multicolumn{2}{|c|}{$M=45,56 ; D E=11,96 ;$ Mín = 24; Máx = 73} \\
\hline Sexo & $\begin{array}{l}\text { Hombre } \\
\text { Mujer }\end{array}$ & $\begin{array}{l}(n=108 ; 36,49 \%) \\
(n=188 ; 63,51 \%)\end{array}$ \\
\hline Zona del país & $\begin{array}{l}\text { Zona Norte Grande } \\
\text { Zona Norte Chico } \\
\text { Región Metropolitana } \\
\text { Zona Central } \\
\text { Zona Sur } \\
\text { Zona Austral } \\
\text { Extranjero }\end{array}$ & $\begin{array}{l}(\mathrm{N}=17 ; 5,74 \%) \\
(\mathrm{N}=1 ; 0,34 \%) \\
(\mathrm{N}=59 ; 19,93 \%) \\
(\mathrm{N}=163 ; 55,07 \%) \\
(\mathrm{N}=51 ; 17,23 \%) \\
(\mathrm{n}=3 ; 1,01 \%) \\
(\mathrm{n}=2 ; 0,68 \%)\end{array}$ \\
\hline $\begin{array}{l}\text { Universidades donde se ha dictado docencia en los } \\
\text { últimos cinco años (en número) }\end{array}$ & $\begin{array}{l}M=1,68 ; D E=1,03 \\
\text { Mín }=1 ; \text { Máx }=7\end{array}$ & \\
\hline $\begin{array}{l}\text { Ejercicio de docencia de pregrado en carreras de la salud } \\
\text { en los últimos } 5 \text { años }\end{array}$ & $\begin{array}{l}\text { En universidades adscritas al CRUCH } \\
\text { En universidades no adscritas al } \mathrm{CRUCH}\end{array}$ & $\begin{array}{l}(\mathrm{n}=243 ; 82,09 \%) \\
(\mathrm{n}=128 ; 43,24 \%)\end{array}$ \\
\hline $\begin{array}{l}\text { Estudios de magíster } \\
\text { Estudios de doctorado }\end{array}$ & $\begin{array}{l}\text { Con estudios de magíster } \\
\text { Sin estudios de magíster } \\
\text { Con estudios de doctorado } \\
\text { Sin estudios de doctorado }\end{array}$ & $\begin{array}{l}(\mathrm{n}=90 ; 30,41 \%) \\
(\mathrm{n}=206 ; 69,59 \%) \\
(\mathrm{n}=220 ; 74,32 \%) \\
(\mathrm{n}=76 ; 25,68 \%)\end{array}$ \\
\hline
\end{tabular}

$\mathrm{N}=$ 296; $\mathrm{M}=$ Media aritmética; $\mathrm{DE}=$ Desviación estándar; Mín = Mínimo; Máx = Máximo; $\mathrm{n}$ = Frecuencia absoluta. 
- El Cuestionario de Prácticas Pedagógicas (CPP) fue elaborado por Pérez y colaboradores ${ }^{12}$ para medir la frecuencia con que el docente realiza una serie de actividades pedagógicas. Presenta 60 afirmaciones sobre estas actividades y el docente debe responder indicando la frecuencia con que las ha realizado, eligiendo una de cinco alternativas (de $0=$ nunca o casi nunca a $4=$ siempre o casi siempre). En académicos de carreras de la salud chilenos fue validado por el mismo autor, encontrándose una estructura de seis factores adecuadamente confiables: Enseñanza centrada en el estudiante, Planificación de la enseñanza, Evaluación del proceso, Relación dialogante, Enseñanza centrada en el profesor y Uso de recursos tecnológicos ${ }^{12}$.

- El Cuestionario de Participación en Actividades de Capacitación en docentes de carreras de la salud (CPAC) fue elaborado por Pérez y colaboradores en el mismo proyecto ${ }^{11-12,15}$. Presenta 15 afirmaciones sobre la frecuencia con que los docentes han realizado actividades de perfeccionamiento disciplinar y pedagógico. El docente debe responder seleccionando una de siete alternativas (de $0=$ nunca a $6=$ siempre). El cuestionario fue validado en docentes de ciencias de la salud de Chile, identificándose 2 factores altamente confiables: Capacitación pedagógica y Capacitación disciplinar ${ }^{11,12,15}$. Adicionalmente, se aplicó un cuestionario sociodemográfico para permitir la descripción muestral.

\section{Procedimiento}

Se contactó a los docentes vía correo electrónico, obteniendo dichas direcciones de bases de dato de acceso público. Adicionalmente, se les pidió a los contactados que redirigieran el correo a los colegas que cumplieran con los criterios de elegibilidad del estudio.

Los docentes debían acceder a la encuesta mediante un hipervínculo en la plataforma y ahí debían aceptar un consentimiento informado para poder acceder a la encuesta. Cada docente podía responder la encuesta sólo una vez.

El procedimiento fue visado por el Comité de Ética de CONICYT.

\section{Análisis de datos}

Se realizó un análisis descriptivo de los factores de cada cuestionario, estimando la confiabilidad de las mediciones mediante alfa de Cronbach, y evaluando su media, desviación estándar, mínimo y máximo. Además se calculó un indicador porcentual (\%) que indicaba el nivel de logro en cada factor en relación al puntaje máximo posible en este $(\%=$ puntaje obtenido/puntaje máximo posible $\left.{ }^{\star} 100\right)$.

La correlación entre los puntajes del CPP y el CPAC se calcularon con el coeficiente de Pearson. Además, se compararon los puntajes del CPP entre los docentes con y sin magíster y con y sin doctorado, aplicando la t de Student para muestra independientes. Por último, se usó regresión lineal múltiple para estimar el efecto sobre las prácticas pedagógicas del perfeccionamiento disciplinar, perfeccionamiento pedagógico, tener magíster y tener doctorado.

Se definió un valor de $\mathrm{p}<0,05$ como estadísticamente significativo.

\section{Resultados}

En el CPP, el indicador porcentual mostró que los docentes realizaban con mayor frecuencia actividades de planificación de la enseñanza $(87,36 \%)$ y relación dialogante $(84,75 \%)$, siendo las menos frecuentes las asociadas a la enseñanza centrada en el profesor $(59,66 \%)$, aunque todos los factores fueron sobre $50 \%$ (Tabla 2).

En el caso del CPAC, los resultados además mostraron una alta participación en actividades de capacitación tanto disciplinar como pedagógica (Tabla 3).

Al evaluar la correlación entre las prácticas pedagógicas (CPP) y la participación en actividades de capacitación disciplinar y pedagógica (CPAC) (Tabla 4), se encontró que la participación en actividades de capacitación disciplinar correlacionó directa y significativamente con todos los factores de prácticas pedagógicas. Esto es, docentes que se perfeccionan más en las temáticas que enseñan realizaron con mayor frecuencia todos los tipos de prácticas pedagógicas evaluadas por el CPP.

En tanto, la participación en actividades de capacitación pedagógica solo se correlacionó con cinco de los factores del CPP, siendo la excepción la enseñanza centrada en el profesor, con el que no hubo una correlación estadísticamente significativa, pudiéndose asumir que ambos fenómenos fueron independientes el uno del otro $(\mathrm{p}=0,25)$. 
Tabla 2. Estadísticos descriptivos del Cuestionario de Prácticas Pedagógicas en docentes de carreras de la salud

\begin{tabular}{|lcccccc|}
\hline Factores del CPP & $\boldsymbol{\alpha}$ & $\mathbf{M}$ & DE & Mín & Máx & \% \\
\hline Enseñanza centrada en el estudiante & 0,85 & 32,79 & 4,77 & 18 & 40 & 68,31 \\
\hline Planificación de la enseñanza & 0,85 & 38,44 & 6,23 & 17 & 48 & 87,36 \\
\hline Evaluación de proceso & 0,81 & 27,42 & 6,58 & 7 & 40 & 68,55 \\
\hline Relación dialogante & 0,82 & 28,12 & 3,36 & 14 & 32 & 84,75 \\
\hline Enseñanza centrada en el profesor & 0,64 & 14,32 & 3,83 & 1 & 24 & 59,66 \\
\hline Uso de recursos tecnológicos & 0,60 & 16,97 & 2,49 & 7 & 20 & 84,85 \\
\hline
\end{tabular}

$\mathrm{n}=296 ;{ }^{*} \mathrm{p}<0,05 ;{ }^{*} \mathrm{p}<0,01 ;{ }^{*} \mathrm{p}<0,001$.

Tabla 3. Estadísticos descriptivos del Cuestionario de Participación en Actividades de Capacitación en docentes de carreras de la salud

\begin{tabular}{|lcccccc|}
\hline Factores del CPAC & $\boldsymbol{\alpha}$ & $\mathbf{M}$ & DE & Mín & Máx & \% \\
\hline Capacitación pedagógica & 0,89 & 36,04 & 8,20 & 11 & 48 & 85,81 \\
\hline Capacitación disciplinar & 0,87 & 30,99 & 4,77 & 6 & 36 & 86,08 \\
\hline
\end{tabular}

$\mathrm{n}=296 ;{ }^{*} \mathrm{p}<0,05 ;{ }^{*} \mathrm{p}<0,01 ;{ }^{*} \mathrm{p}<0,001$.

Tabla 4. Correlación de Pearson entre la participación en actividades de capacitación disciplinar y pedagógica y las prácticas pedagógicas

\begin{tabular}{|lcc|}
\hline Factores del CPP & Capacitación pedagógica & Capacitación disciplinar \\
\hline Enseñanza centrada en el estudiante & $0,35^{* * *}$ & $0,31^{* * *}$ \\
\hline Planificación de la enseñanza & $0,43^{* * *}$ & $0,22^{* * *}$ \\
\hline Evaluación de proceso & $0,48^{* * *}$ & $0,17^{* *}$ \\
\hline Relación dialogante & $0,34^{* * *}$ & $0,27^{* * *}$ \\
\hline Enseñanza centrada en el profesor & $-0,07$ & $0,18^{* *}$ \\
\hline Uso de recursos tecnológicos & $0,20^{* * *}$ & $0,21^{* * *}$ \\
\hline
\end{tabular}

$n=296 ;{ }^{*} p<0,05 ;{ }^{*} p<0,01 ;{ }^{*} p<0,001$.

Posteriormente, se compararon los puntajes de las prácticas pedagógicas entre quienes presentaban y no presentaban estudios de postgrado (Tabla 5).

En el caso de los estudios de magíster, quienes poseían estos estudios presentaban puntajes significativamente superiores a quienes no los presentaban, solo en los factores de planificación de la enseñanza $(\mathrm{p}<0,05)$ y evaluación de proceso $(\mathrm{p}<0,05)$. Sin embargo, en el caso de los estudios de doctorado, no se encontró diferencias estadísticamente significativas en las prácticas pedagógicas de quienes los poseían y quienes no.
Por último, se buscó establecer la capacidad predictiva de la participación en perfeccionamiento disciplinar y docente, y los estudios de postgrado sobre los puntajes obtenidos en los seis factores de prácticas pedagógicas medidos con el CPP. Para esto, se desarrollaron seis modelos de regresión lineal múltiple. En todos estos, como predictores se consideró la participación en actividades de capacitación pedagógica y de capacitación disciplinar, medidas con el CPAC, el tener estudios de magíster (donde $1=$ Posee y $0=$ No posee) y el tener estudios de doctorado (donde $1=$ Posee y $0=$ No posee). 
Tabla 5. Comparación de las prácticas pedagógicas entre los docentes que poseen y no poseen estudios de magíster y doctorado

\begin{tabular}{|c|c|c|c|c|c|c|c|c|c|c|}
\hline \multirow[b]{3}{*}{ Factores del CPP } & \multicolumn{4}{|c|}{ Magíster } & \multicolumn{6}{|c|}{ Doctorado } \\
\hline & \multicolumn{2}{|c|}{ Sin magíster } & \multicolumn{2}{|c|}{ Con magíster } & & \multicolumn{2}{|c|}{ Sin doctorado } & \multicolumn{2}{|c|}{ Con doctorado } & \multirow[b]{2}{*}{$\mathbf{t}$} \\
\hline & M & DE & $\mathbf{M}$ & DE & & M & DE & M & DE & \\
\hline $\begin{array}{l}\text { Enseñanza centrada en el } \\
\text { estudiante }\end{array}$ & 32,17 & 4,42 & 33,07 & 4,90 & $-1,50$ & 32,83 & 4,85 & 32,70 & 4,56 & 0,20 \\
\hline Planificación de la enseñanza & 37,42 & 6,35 & 38,89 & 6,18 & $-1,86^{\star}$ & 38,39 & 6,32 & 38,59 & 6,11 & $-0,24$ \\
\hline Evaluación de proceso & 25,38 & 6,90 & 28,31 & 6,24 & $-3,59 * * *$ & 27,44 & 6,41 & 27,36 & 7,08 & 0,09 \\
\hline Relación dialogante & 27,64 & 3,58 & 28,33 & 3,25 & $-1,62$ & 28,06 & 3,31 & 28,30 & 3,34 & $-0,54$ \\
\hline $\begin{array}{l}\text { Enseñanza centrada en el } \\
\text { profesor }\end{array}$ & 14,79 & 3,65 & 14,12 & 3,89 & 1,38 & 14,25 & 4,01 & 14,55 & 3,25 & $-0,60$ \\
\hline Uso de recursos tecnológicos & 16,56 & 2,62 & 17,16 & 2,42 & $-1,91$ * & 17,03 & 2,55 & 16,80 & 2,33 & 0,69 \\
\hline
\end{tabular}

$\mathrm{n}=296 ;{ }^{*} \mathrm{p}<0,05 ;{ }^{*} \mathrm{p}<0,01 ;{ }^{*} \mathrm{p}<0,001$.

Como variables dependientes se consideró cada factor del CPP:

\section{Enseñanza centrada en el estudiante}

Se encontró que los cuatro predictores se asociaban de manera estadísticamente significativa con este, dando cuenta de $15,63 \%$ de su variación $(\mathrm{p}<0,001)$. Individualmente, se encontró que una enseñanza más frecuentemente centrada en el estudiante era predicha por una mayor participación en actividades de capacitación pedagógica $(p<0,001)$, y una mayor participación en actividades de capacitación disciplinar $(\mathrm{p}<0,001)$, explicando $5,49 \%$ y $3,87 \%$ de esta, respectivamente (Tabla 6).

\section{Planificación de la enseñanza}

El conjunto de predictores se asociaron de manera estadísticamente significativa con este factor, dando cuenta de $18,08 \%$ de su variación $(\mathrm{p}<0,001)$. Individualmente, una mayor implicación en actividades de planificación de la enseñanza estuvo asociada a una mayor participación en actividades de capacitación pedagógica ( $\mathrm{p}<0,001)$, que explicaba $12,65 \%$ de la variable (Tabla 7).

\section{Evaluación de proceso}

Los cuatro predictores se asociaron de manera estadísticamente significativa con este factor, dando cuenta de $25,09 \%$ de su variación $(\mathrm{p}<0,001)$. Individualmente, una evaluación de proceso más frecuente por parte del profesor ocurría cuando
Tabla 6. Resultados de la regresión lineal múltiple del efecto de la participación en actividades de capacitación y formación de postgrados sobre la Enseñanza centrada en el estudiante

\begin{tabular}{|lcccc|}
\hline Predictores & B & EE & $\boldsymbol{\beta}$ & sr $^{\mathbf{2}}$ \\
Constante & 20,00 & & & \\
\hline Capacitación pedagógica & $0,15^{* * *}$ & 0,03 & 0,26 & 0,05 \\
\hline Capacitación disciplinar & $0,22^{* * *}$ & 0,06 & 0,22 & 0,04 \\
Estudios de magíster & 0,86 & 0,57 & 0,08 & 0,01 \\
\hline Estudios de doctorado & $-0,36$ & 0,60 & $-0,03$ & $<0,01$ \\
\hline
\end{tabular}

$R^{2}=0,17^{* *} ; R^{2}$ ajustado $=0,16 . B=$ coeficientes de regresión no estandarizados; $\mathrm{EE}=$ errores estándar; $\beta=$ coeficientes de regresión estandarizados; $\mathrm{sr}^{2}=$ correlaciones semiparciales al cuadrado; $\mathrm{R}^{2}=$ coeficiente de determinación; $R^{2}=$ coeficiente de determinación ajustado. $n=296$; ${ }^{*} p<0,05 ;{ }^{*} p<0,01 ;{ }^{*} p<0,001$.

este tenía una mayor participación en actividades de capacitación pedagógica $(\mathrm{p}<0,001)$, y cuando tenía estudios de magíster $(\mathrm{p}<0,01)$, factores que explicaban $17,96 \%$ y $2,85 \%$ de la variación de este factor respectivamente (Tabla 8 ).

\section{Relación dialogante}

Los predictores se asociaban de manera estadísticamente significativa con este factor, dando cuenta de $13,17 \%$ de su variación $(\mathrm{p}<0,001)$. Individualmente, los docentes que más establecían este tipo de relación eran quienes participaban en más actividades, tanto de capacitación pedagógica $(\mathrm{p}<0,001)$, como de capacitación disciplinar 
Tabla 7. Resultados de la regresión lineal múltiple del efecto de la participación en actividades de capacitación y formación de postgrados sobre la planificación de la enseñanza

\begin{tabular}{|lcccc|}
\hline Predictores & B & EE & $\boldsymbol{\beta}$ & $\mathbf{s r}^{\mathbf{2}}$ \\
\hline Constante & 23,95 & & & \\
\hline Capacitación pedagógica & $0,30 * * *$ & 0,04 & 0,39 & 0,13 \\
\hline Capacitación disciplinar & 0,09 & 0,08 & 0,07 & $<0,01$ \\
\hline Estudios de magíster & 1,18 & 0,73 & 0,09 & 0,01 \\
\hline Estudios de doctorado & 0,40 & 0,78 & 0,03 & $<0,01$ \\
\hline
\end{tabular}

$R^{2}=0,19^{* * *} ; R^{2}$ ajustado $=0,18 . B=$ coeficientes de regresión no estandarizados; $\mathrm{EE}=$ errores estándar; $\beta=$ coeficientes de regresión estandarizados; $\mathrm{sr}^{2}=$ correlaciones semiparciales al cuadrado; $R^{2}=$ coeficiente de determinación; $R^{2}=$ coeficiente de determinación ajustado. $n=296$; ${ }^{*} p<0,05 ;{ }^{*} p<0,01 ;{ }^{*} p<0,001$.

Tabla 9. Resultados de la regresión lineal múltiple del efecto de la participación en actividades de capacitación y formación de postgrados sobre la relación dialogante

\begin{tabular}{|lcccc|}
\hline Predictores & B & EE & $\boldsymbol{\beta}$ & $\mathbf{s r}^{2}$ \\
\hline Constante & 20,06 & & & \\
\hline Capacitación pedagógica & $0,11 * * *$ & 0,02 & 0,27 & 0,06 \\
\hline Capacitación disciplinar & $0,12^{* *}$ & 0,04 & 0,16 & 0,02 \\
\hline Estudios de magíster & 0,67 & 0,40 & 0,09 & 0,01 \\
\hline Estudios de doctorado & 0,18 & 0,43 & 0,02 & $<0,01$ \\
\hline
\end{tabular}

$R^{2}=0,14^{* * *} ; R^{2}$ ajustado $=0,13 B=$ coeficientes de regresión no estandarizados; $\mathrm{EE}=$ errores estándar; $\beta=$ coeficientes de regresión estandarizados; $\mathrm{sr}^{2}=$ correlaciones semiparciales al cuadrado; $R^{2}=$ coeficiente de determinación; $R^{2}=c o-$ eficiente de determinación ajustado. $n=296 ;{ }^{*} p<0,05$; ${ }^{*} p<0,01 ;{ }^{*} p<0,001$.

$(\mathrm{p}<0,001)$. Estos factores explicaban 5,83\% y $2,15 \%$ de la variación de este factor, respectivamente (Tabla 9).

\section{Enseñanza centrada en el profesor}

El conjunto de predictores se asociaron de manera estadísticamente significativa con este factor, dando cuenta de 4,29\% de su variación $(\mathrm{p}<0,001)$. Individualmente, se observó que la enseñanza centrada en el profesor era más frecuente en docentes con una menor participación en actividades de capacitación pedagógica $(\mathrm{p}<0,05)$, y una mayor participación en actividades de capa-
Tabla 8. Resultados de la regresión lineal múltiple del efecto de la participación en actividades de capacitación y formación de postgrados sobre la evaluación de proceso

\begin{tabular}{|lcccc|}
\hline Predictores & B & EE & $\boldsymbol{\beta}$ & $\mathbf{s r}^{\mathbf{2}}$ \\
Constante & 11,94 & & & \\
Capacitación pedagógica & $0,38^{* * *}$ & 0,04 & 0,47 & 0,18 \\
\hline Capacitación disciplinar & $<0,01$ & 0,08 & $<0,01<0,01$ \\
\hline Estudios de magíster & $2,46^{* *}$ & 0,73 & 0,17 & 0,03 \\
\hline Estudios de doctorado & 0,55 & 0,78 & $0,04<0,01$ \\
\hline
\end{tabular}

$R^{2}=0,26^{* * *} ; R^{2}$ ajustado $=0,25 . B=$ coeficientes de regresión no estandarizados; $\mathrm{EE}=$ errores estándar; $\beta=$ coeficientes de regresión estandarizados; $\mathrm{sr}^{2}=$ correlaciones semiparciales al cuadrado; $R^{2}=$ coeficiente de determinación; $R^{2}=$ coeficiente de determinación ajustado. $n=296$; ${ }^{*} p<0,05 ;{ }^{*} p<0,01 ;{ }^{*} p<0,001$.

Tabla 10. Resultados de la regresión lineal múltiple del efecto de la participación en actividades de capacitación y formación de postgrados sobre la enseñanza centrada en el profesor

\begin{tabular}{|lcccc|}
\hline Predictores & B & EE & $\boldsymbol{\beta}$ & sr $^{\mathbf{2}}$ \\
\hline Constante & 11,38 & & & \\
\hline Capacitación pedagógica & $-0,07^{*}$ & 0,03 & $-0,16$ & 0,02 \\
\hline Capacitación disciplinar & $0,19 * * *$ & 0,05 & 0,24 & 0,05 \\
\hline Estudios de magíster & $-0,39$ & 0,48 & $-0,05$ & $<0,01$ \\
\hline Estudios de doctorado & $-0,20$ & 0,51 & $-0,02$ & $<0,01$ \\
\hline
\end{tabular}

$R^{2}=0,06^{* * *} ; R^{2}$ ajustado $=0,04 . B=$ coeficientes de regresión no estandarizados; $E E=$ errores estándar; $\beta=$ coeficientes de regresión estandarizados; $\mathrm{sr}^{2}=$ correlaciones semiparciales al cuadrado; $R^{2}=$ coeficiente de determinación; $R^{2}=c o-$ eficiente de determinación ajustado. $n=296$; ${ }^{*} p<0,05$; ${ }^{*} p<0,01 ;{ }^{*} p<0,001$

citación disciplinar $(\mathrm{p}<0,001)$, explicando 2,06\% y $4,52 \%$ de la variación de este factor, respectivamente (Tabla 10).

\section{Uso de recursos tecnológicos}

Los cuatro predictores se asociaron de manera estadísticamente significativa con este factor, dando cuenta de $6,41 \%$ de su variación $(\mathrm{p}<0,001)$. Individualmente, resultó que el uso de recursos tecnológicos era más frecuente en los docentes con mayor participación en actividades de capacitación disciplinar $(\mathrm{p}<0,001)$, lo que daba cuenta de 2,58\% de la variación de este factor (Tabla 11). 
Tabla 11. Resultados de la regresión lineal múltiple del efecto de la participación en actividades de capacitación y formación de postgrados sobre el Uso de recursos tecnológicos

\begin{tabular}{|c|c|c|c|c|}
\hline Predictores & B & EE & $\boldsymbol{\beta}$ & $s r^{2}$ \\
\hline Constante & 12,37 & & & \\
\hline Capacitación pedagógica & 0,04 & 0,02 & 0,12 & 0,01 \\
\hline Capacitación disciplinar & $0,09 * *$ & 0,03 & 0,18 & 0,03 \\
\hline Estudios de magíster & 0,60 & 0,31 & 0,11 & 0,01 \\
\hline Estudios de doctorado & $-0,31$ & 0,33 & $-0,05$ & $<0,01$ \\
\hline \multicolumn{5}{|c|}{$\begin{array}{l}R^{2}=0,08^{* * *} ; R^{2} \text { ajustado }=0,06 . B=\text { coeficientes de regresión } \\
\text { no estandarizados; } E E=\text { errores estándar; } \beta=\text { coeficientes } \\
\text { de regresión estandarizados; } s r^{2}=\text { correlaciones semiparcia- } \\
\text { les al cuadrado; } R^{2}=\text { coeficiente de determinación; } R^{2}=c 0- \\
\text { eficiente de determinación ajustado. } n=296 ;{ }^{*} p<0,05 ; \\
{ }^{*} p<0,01 ;{ }^{*} p<0,001 \text {. }\end{array}$} \\
\hline
\end{tabular}

\section{Discusión}

Los resultados muestran que las prácticas pedagógicas más frecuentes son aquellas asociadas a modelos curriculares que se promueven en la actualidad en carreras de la salud, esto es: enseñanza centrada en el estudiante, planificación de la enseñanza, evaluación de proceso, relación dialogante y uso de recursos tecnológicos ${ }^{12,19-20}$. En tanto, la enseñanza centrada en el profesor presentó el menor nivel de los factores evaluados. Se debe tener en consideración que este factor se relaciona a un modelo de educación tradicional ${ }^{4-5}$, modelo ampliamente cuestionado, que, aunque se propone que sea reemplazado por un modelo constructivista centrado en el aprendizaje significativo ${ }^{21-23}$, sigue siendo muy común en las universidades latinoamericanas ${ }^{24}$.

La planificación de la enseñanza, una de las competencias más importantes ${ }^{25}$, alcanzó un porcentaje cercano a $90 \%$. Al respecto, De Miguel indica que la calidad de la enseñanza, más que del conocimiento del profesor, depende de lo que éste planifica en función de las necesidades y posibilidades de sus alumnos y de lo que hace para ofrecerles oportunidades para el aprendizaje ${ }^{26}$.

Respecto a la participación en actividades de capacitación disciplinar y pedagógica, ambos presentaron un alto nivel de logro (sobre $80 \%$ ). Escenario recomendable, según instituciones internacionales como UNESCO ${ }^{27}$, que sugiere es- tablecer como política esencial de las instituciones terciarias la formación de sus docentes.

En cuanto a las correlaciones entre las prácticas pedagógicas y la participación en actividades de capacitación, se encontró que una mayor capacitación disciplinar se asociaba a una mayor frecuencia de todas las prácticas pedagógicas. Esto sería esperable, pues los docentes con mayor experiencia y capacitación en una determinada disciplina logran valorar de mejor manera aquellos aspectos más relevantes de la temática, centrando la docencia en aspectos fundamentales, como la planificación y evaluación del proceso ${ }^{28}$.

Específicamente, que los docentes que se capacitan más en sus disciplinas realicen con mayor frecuencia una enseñanza centrada en el profesor, podría explicarse porque un mayor nivel de dominio y especialización disciplinar puede traducirse en una mayor tendencia a recurrir a instancias que permitan transmitir el conocimiento adquirido, considerando que la docencia tradicional puede ser ampliamente eficiente y no necesariamente negativa, si es bien usada ${ }^{29}$.

Por su parte, la participación en actividades de capacitación pedagógica se correlacionó significativamente con los cinco factores relacionados a un modelo de educación constructivista. Según Pérez, cuando el docente participa en buenos programas de capacitación pedagógica, esto le permite, no sólo organizar y fundamentar las prácticas que intuitivamente realizan, además, los docentes son motivados a seguir especializándose en educación, generan una visión más amplia de su rol y comprenden mejor el proceso enseñanza-aprendizaje $^{15}$. Los estudios muestran que capacitarse en planificación, estrategias de enseñanza, manejo de aula y evaluación, conducen a una transformación en la forma de hacer docencia ${ }^{15-16}$.

En cuanto a las diferencias de las prácticas entre quienes poseen y no poseen estudios de postgrado, los resultados indicaron que quienes poseen estudios de magíster presentaban puntajes significativamente superiores a quienes no lo presentaban en los factores planificación de la enseñanza y evaluación de proceso de prácticas pedagógicas. Al respecto, Lara encontró un crecimiento personal y profesional en los participantes de estudios de magíster, quienes logran comprender la interacción entre la teoría y la práctica, y al mismo tiempo alcanzan mayor independencia del ejercicio de la profesión docente ${ }^{30}$. 
No hubo diferencias asociadas al doctorado. Es necesario considerar que este grado forma personas capaces de desarrollar investigaciones de manera independiente. Sin embargo, aunque la investigación es la que concentra recursos, recompensas y reconocimiento en la universidad, no está directamente asociada a la labor docente ${ }^{31}$.

Respecto a identificar el efecto conjunto de la formación de postgrado y la participación en actividades de capacitación disciplinar y pedagógica sobre las prácticas pedagógicas en docentes de carreras de la salud, podemos decir que las formas de capacitación docente evaluadas sí afecta la práctica pedagógica, en todos los factores. De hecho, afectan entre 4,29\% (enseñanza centrada en el profesor) y $25,09 \%$ (en evaluación de proceso), lo que muestra que aunque es un factor multideterminado, el invertir en capacitación para los académicos puede tener efectos beneficiosos en su quehacer. Aunque es necesario considerar que el efecto individual no fue el mismo, de modo que capacitarse en pedagogía estaba asociada a una mayor frecuencia de actividades propias de la enseñanza centrada en el estudiante, planificación de la enseñanza, evaluación de proceso y relación dialogante, todos aspectos promovidos por el constructivismo, modelo imperante en las capacitaciones. Contrariamente, esta forma de capacitación se asoció a un menor uso de la enseñanza centrada en el profesor, propia del paradigma tradicional y conceptualmente opuesta a lo que ofrecen las capacitaciones educativas ${ }^{4,15-16}$. En tanto, la capacitación disciplinar se asoció a un mayor uso de la enseñanza centrada en el profesor y en el estudiante, de relación dialogante y de recursos tecnológicos, lo que es coherente con la concepción tradicional de que un docente debe ser especialistas del tema que enseña ${ }^{4-7}$. Por último, los estudios de magíster afectan la evaluación de proceso, esperable si se asume que un mayor dominio técnico de lo enseñado a través de la maestría puede permitir entender nuevas y más pertinentes formas de evaluarlo ${ }^{30}$.

\section{Referencias}

1. Araneda-Guirriman C, Rodríguez-Ponce E, PedrajaRejas L. Relación entre el financiamiento fiscal, la calidad del cuerpo académico y la retención de estudiantes universitarios en Chile. Formación universitaria 2013; 6(6): 55-64.

2. Brunner J, Ganga F. Reflexiones en torno a economía política y gobernanza de los sistemas nacionales e instituciones de educación superior en América Latina. Interciencia; 41: 573-9.

3. Stenfors-Hayes T, Hult H, Dahlgren LO. Three ways of understanding development as a teacher. Eur J Dent Edu 2012; 16 (1): e151-7.

4. Fasce E. ¿Son suficientes las competencias profesionales en la enseñanza de las ciencias de la salud? Rev Educ Cienc Salud 2008; 5 (2): 81-0.

5. Harden R, Crosby R. The good teacher is more than a lecturer-the twelve roles of the teacher. AMEE Guide No 20. Med Teach 2000; 22 (4): 334-47.

6. Syed Z, Mahboob U, Wajid G, Iqbal Z. Medical Teachers Awareness and Prioriti- zation of their Roles. Adv Health Prof Educ 2015; 1 (2): 49-54.

7. Snider V, Roehl R. Teachers' beliefs about pedagogy and related issues. Psychol Schools 2007; 44 (8): 873-86.

8. Espejo R. El desarrollo de profesores universitarios y escolares en Chile: ¿Brecha o Continuidad?. Perspectiva Educacional 2014; 53 (2): 3-19.

9. Díaz V. Formación docente, práctica pedagógica y saber pedagógico. Laurus 2006; 12: 88-103.

10. Cañedo T, Figueroa A. La práctica docente en educación superior: una mirada hacia su complejidad. Sinéctica 2013; 41: 2-18.

11. Carrasco C, Pérez C, Torres G, Fasce E. Relación entre prácticas pedagógicas y estrategias de aprendizaje en docentes de carreras de la salud. Rev Med Chile 2016; 144 (9): 1199-206.

12. Pérez C, Vaccarezza G, Aguilar C, Coloma K. Cuestionario de prácticas pedagógicas: análisis de su estructura factorial y consistencia interna en docentes de carreras de la salud. Rev Med Chile 2016; 144: 795-805.

13. Camargo M, Vergara M, Calvo G, Londoño M, Franco $\mathrm{N}$, Zapata $\mathrm{F}$, et al. Las necesidades de formación permanente del docente. Educ educ 2004; 7: 79-112.

14. Zabalza M, Cid A, Trillo F. Formación docente del profesorado universitario. El difícil tránsito a los enfoques institucionales. Revista Española de Pedagogía 2014; 72: 39-54.

15. Pérez C, Fasce E, Coloma K, Vaccarezza G, Ortega J. Percepción de académicos de carreras de la salud de Chile sobre el perfeccionamiento docente. Rev Med Chile 2013; 141: 787-92.

16. Triviño X, Sirhan M, Moore P, Montero L. Impacto de un programa de formación en docencia en una escuela de medicina. Rev Med Chile 2011; 139: 1508-15.

17. Vivas M, Becerra G, Díaz D. Propuesta para la for- 
mación del profesorado universitario. Revista Acción Pedagógica. 2003; 12 (2): 60-76.

18. Zabalza M. Ser profesor universitario hoy. La Cuestión Universitaria 2009; 5: 69-81.

19. Srinivasan M, Li S, Meyers F, Pratt D, Collins J, Braddock $\mathrm{C}$, et al. Teaching as a compentency: Competencies for medical educator. Acad Med 2011; 86 (10): 1211-20.

20. Robles F, Jiménez A, Orazco V. Las Competencias del Profesor Universitario. Educateconciencia 2016; 4 (4): 201-6.

21. Castilla M, López C. Los roles del docente en la educación médica. Educ. Educ 2007; 10: 105-13.

22. Rosenbaum M, Ferguson K, Lenoch S. A teaching scholars program to improve faculty development. Acad Med 2001; 76: 572-3.

23. Slotnick H B. How doctors learn: education and learning across the medical-school-to-practice trajectory. Acad Med 2001; 76: 1013-26.

24. Morales I, Borroto R, Fernández B. Políticas y estrategias para la transformación de la educación superior en América Latina y el Caribe. Educ Méd Super 2005; 19 (1): 1-11.

25. Álvarez V, Asencio I, Clares J. Perfiles docentes para el espacio europeo de educación superior (EEES) en el ámbito universitario español. Revista electrónica de investigación y evaluación educativa 2009; 15 (1): 1-18.

26. De Miguel, M. Metodologías para optimizar el aprendizaje. Segundo objetivo del Espacio Europeo de Educación Superior. Revista Interuniversitaria de Formación del Profesorado 2006; 20 (3): 71-91.

27. UNESCO. Declaración mundial sobre la educación superior en el siglo XXI: visión y acción. Conferencia Mundial sobre Educación Superior. París: UNESCO; 1998. http://www.unesco.org/education/educprog/wche/ declaration_spa.htm.

28. De Vincenzi A. La formación pedagógica del profesor universitario. Un desafío para la reflexión y revisión de la práctica docente en el nivel superior. Aula 2012; 18 : 111-22.

29. Coppola B. The distinctiveness of higher education. J Chem Educ 2013; 90: 955-6.

30. Lara L, Sánchez L, Bravo G. El desarrollo profesional de los maestrantes del programa de la Maestría en Educación de la Universidad de Cienfuegos, Cuba. Revista Iberoamericana de Educación 2012; 58 (1): 1-13.

31. Fook CY. Best practices of teaching in higher education in United States: a case study. Procedia Soc Behav Sci 2012; 40: 4817-21. 